\title{
FERRAMENTAS E TÉCNICAS PARA A AVALIAÇÃO DE PROJETOS DE INOVAÇÃO RADICAL OU DE GRANDES INCERTEZAS: UMA REVISÃO INTEGRATIVA DE LITERATURA
}

\author{
Robériton Luís Oliveira Ribeiro1; Ingrid Winkler²; Carlos César Ribeiro Santos ${ }^{3}$ \\ 1 SENAI - CIMATEC; Salvador/Bahia; roberiton@gmail.com \\ 2 SENAI - CIMATEC; Salvador/Bahia \\ ${ }^{3}$ SENAI - CIMATEC; Salvador/Bahia
}

\begin{abstract}
Resumo: Inovação é importante para as empresas se manterem competitivas no mercado. Várias definições sobre inovação são encontradas na literatura científica e profissional, o que evidencia visões conflitantes quanto ao seu conceito. O objetivo deste trabalho é caracterizar o conhecimento atual sobre quais ferramentas e técnicas de avaliação de investimentos são aplicáveis à avaliação de projetos de inovação radical (RI) ou de grandes incertezas. Como procedimento metodológico, foi realizada uma revisão integrativa de literatura. As publicações foram selecionadas através de critérios de classificação, exclusão e qualificação. Como resultado, identificou-se que a abordagem opções reais (RO) é uma alternativa às técnicas tradicionais de avaliação de investimento para projetos de RI ou de grandes incertezas.
\end{abstract}

Palavras-Chave: inovação radical; inovação contínua, avaliação de investimentos; opção real.

\section{TOOLS AND TECHNIQUES FOR VALUATION OF RADICAL INNOVATION PROJECTS OR WITH HIGH LEVEL OF UNCERTAINTY: AN INTEGRATIVE LITERATURE REVIEW}

\begin{abstract}
Innovation is essential for the company's competitiveness. There are several definitions about innovation that are known in the scientific and professional literature, which shows conflicting views regarding its concept. The goal of this paper is, through an integrative literature review, identify which tools and techniques is applicable for valuation of radical innovation (RI) projects or with high level of uncertainty. The publications were selected by classification, exclusion and qualification criteria. As a result, the real option (RO) approach has been found as an alternative to traditional investment valuation techniques for RI projects or with high level of uncertainty.
\end{abstract}

Keywords: radical innovation, continuous innovation, investment valuation; real option. 


\section{INTRODUÇÃO}

A globalização possibilita a concorrência empresarial em amplitude global [1]. Este cenário exige que as empresas, para atenderem aos padrões de excelência do mercado e manterem-se competitivas, se reinventem, sejam mais eficientes e continuamente invistam na melhoraria de qualidade de seus produtos e serviços [2] e a inovação é um fator importante para que se mantenham competitivas no mercado. Inovação Continuada é um processo contínuo de construção e formação de capacidades de inovação incremental ou radical para aumentar o potencial de uma empresa na geração de desempenho inovador [1].

Apesar da onipresença do termo "inovação", há diferentes definições de seu conceito, principalmente quanto aos tipos de inovação. Para [3], criador da teoria de inovação disruptiva, o termo "inovação disruptiva", por exemplo, está sendo mal compreendido por pesquisadores, escritores e consultores que aplicam o termo a qualquer situação que abale uma indústria. A inovação incremental é aquela que melhora produtos ou serviços estabelecidos, junto com as dimensões de desempenho que os clientes habituais valorizam, já a inovação radical, apesar de dar suporte à melhoria de desempenho percebido pelo cliente, é proveniente de tecnologias que descontinuam outras [4]. A inovação disruptiva ou que causa ruptura, emergem de produtos de pior desempenho, pelo menos a curto prazo, e que trazem ao mercado uma proposição de valor muito diferente daquela disponível até então e que, em algum momento, provocarão o fracasso de empresas líderes de mercado [4]. Desta forma, nem toda RI causa disrupção de empresas líderes ou estabelecidas no mercado.

A Gestão de Portfólio de Projetos é uma disciplina que visa ajudar as empresas a planejarem e melhor aplicarem seus investimentos e esforços através de processos estruturados que visam, de modo geral, identificar, selecionar e priorizar, de acordo com critérios de avaliação, a execução de projetos que trarão maiores benefícios para a empresa [5]. Devido à competitividade do mercado, é importante minimizar os riscos de investimentos em projetos que não trarão benefícios para a empresa [6]. [7] acrescenta que é necessário ter cuidado com os projetos de inovações revolucionárias, no sentido de que aplicar metodologias de previsão para avaliar projetos de RI tem se mostrado ineficazes. Enquanto a inovação incremental pode ser fundamentada em dados históricos e tecnologias conhecidas [8] a RI em empresas estabelecidas é um empreendimento arriscado, exacerbado por altos níveis de incerteza $[8,9]$.

Neste contexto, este estudo tem como objetivo identificar quais ferramentas e técnicas de avaliação de investimentos são aplicáveis à avaliação de projetos de inovação radical ou de grandes incertezas.

O trabalho está organizado em quatro seções: além desta introdução, a seção 2 descreve a metodologia utilizada na investigação; a seção 3 aborda os resultados e discute-os, na seção 4 são apresentadas as conclusões e indicações de pesquisas futuras. 


\section{METODOLOGIA}

Este trabalho, que é uma revisão integrativa de literatura que visa identificar, avaliar e sintetizar informações relevantes [10], consistiu nas seguintes etapas: estratégia de pesquisa, seleção do material de estudo, refinamento da seleção, síntese da extração de dados e resultados.

\subsection{Estratégia de Pesquisa}

Inicialmente, a estratégia consistiu na realização de uma busca na base de dados Scopus com os termos principais relacionados à identificação da pesquisa. Os termos foram utilizados em inglês na seção de busca com a seguinte expressão: valuation of radical innovation projects. O resultado desta busca resultou em apenas 05 publicações. Diante disso, uma nova busca, usando termos menos específicos, foi realizada com a seguinte expressão: valuation of innovation projects, que resultou na identificação de 163 trabalhos. Os mesmos critérios foram aplicados na base de dados Web of Science e os seguintes resultados foram obtidos: 05 artigos para a expressão valuation of radical innovation projects e 145 para a expressão valuation of innovation projects. As buscas foram realizadas no dia 27 de julho de 2019.

\subsection{Seleção do Material de Estudo}

Os critérios (C) utilizados para selecionar o material de estudo foram: $\mathrm{C}$ : materiais publicados entre os anos de 2017 e 2019; C2: publicados nos idiomas inglês, português ou espanhol; C3: exclusão de publicações repetidas; C4: exclusão de publicações das áreas de saúde e enfermagem e C5: publicações de acesso gratuito e que no resumo era possível identificar a aderência quanto à identificação da pesquisa. Ao aplicar o $\mathrm{C} 1$ de seleção em ambas as bases, o material de estudo foi reduzido de 318 a 77, sendo 36 da base Scopus e 41 da base Web of Science. Ao aplicar o C2, a quantidade se manteve. Como as buscas foram iniciadas na base Scopus, a preferência foi manter as publicações desta base e excluir as da base Web of Science. O C3 resultou na exclusão de 18 publicações, sendo 01 da base Scopus e 17 da base Web of Science, reduzindo para 59 publicações. Após aplicação do C4, outras 02 publicações foram excluídas, ambas da base Scopus. Após aplicação do último critério de seleção, a quantidade de artigos foi reduzida a 12 , sendo 08 da base de dados Scopus e 04 da base Web of Science.

\subsection{Refinamento da Seleção}

Para garantir a qualidade e credibilidade desta revisão de literatura, as publicações de acesso gratuito foram lidas e avaliadas quanto ao grau de aderência 
aos seguintes critérios de qualidade, fundamentados de acordo com respostas a duas perguntas: Q1 - a publicação aborda o tema principal desta pesquisa, avaliação da inovação e projetos de grandes incertezas? Q2 - a publicação aborda sobre técnicas e ferramentas de avaliação de projetos de inovação radical ou de grandes incertezas? Para cada publicação lida, pontuações foram definidas de acordo com a escala Likert de pontuação mínima $0(\mathrm{P} 0)$ e máxima $3(\mathrm{P} 3)$, onde 0 equivale a não tem relação e 3 significa alta relação, conforme tabela 1. Publicações em que a soma das pontuações Q1 e Q2 resultou numa média igual ou inferior a 1 foram desconsideradas.

Tabela 1. Escala de avaliação de qualidade

\begin{tabular}{|c|c|c|c|c|c|}
\hline Perguntas & P0 & P1 & P2 & P3 & Média Descarte \\
\hline Q1 & Não & Baixa & Média & Alta & $<=1$ \\
\hline Q2 & Não & Baixa & Média & Alta & $<$ \\
\hline
\end{tabular}

Fonte: Próprio Autor (2019)

Após a verificação de qualidade, 01 artigo da base Web of Science foi excluído, totalizando 11 artigos qualificados para análise e extração de dados.

\subsection{Síntese da Extração de Dados}

Esta etapa consiste em apresentar, de forma sucinta, as etapas executadas a fim de obter o montante de publicações qualificadas para extração e análise de dados, além de direcionar o desenvolvimento de pesquisas futuras e complementares a esta. A sequência abaixo apresenta os critérios de busca de literatura, assim como os processos de avaliação e seleção final do portfólio de publicações para extração e análise de dados.

1. Identificação da pesquisa

2. Estratégia de pesquisa: 318 publicações encontradas
a. Data de busca: 27 de Julho de 2019
b. Base de dados: Scopus e Web of Science
c. Operação booleana 1: valuation of radical innovation projects
d. Operação booleana 2: Valuation of innovation projects

3. Seleção do material de estudo: 12 publicações selecionadas
a. C1 - exclusão de 241 publicações
b. C2 - não houve exclusão
c. C3 - exclusão de 18 publicações
d. C4 - exclusão de 02 publicações
e. C5 - exclusão de 45 publicações

4. Refinamento da seleção: 11 artigos qualificados

a. Indicador de descarte: $<=1$ 


\section{RESULTADOS E DISCUSSÃO}

Esta seção apresenta algumas citações que indicam ferramentas e técnicas aplicáveis à avaliação de projetos de RI ou de grandes incertezas, assim como alguns indicadores sobre as 11 publicações qualificadas.

\subsection{Ferramentas e Técnicas Utilizadas}

As citações a seguir apresentam, de forma branda, a ferramenta, técnica ou abordagem mencionada em cada artigo qualificado como aplicáveis à avaliação de projetos de RI ou de grandes incertezas.

RO é uma ferramenta adequada para avaliar de forma apropriada os riscos e recompensas de um projeto, possibilitando tomadas de decisão estratégicas que podem aumentar retornos e diminuir perdas sobre projetos de alto grau de incertezas [11]. O desenvolvimento de uma startup ou uma nova droga, por exemplo, envolve investir uma quantidade incerta de capital e de tempo para trazer ao mercado um produto cujas características de desempenho ou qualidade são incertas. RO é uma ferramenta poderosa para avaliar projetos incertos e de flexibilidade intrínseca [12].

Técnicas tradicionais de avaliação de investimentos, como Valor Presente Líquido (VPL) e árvore de decisão, são limitadas quando os custos esperados e fluxos de caixa são incertos. RO tem ajudado acadêmicos e gerentes a melhor avaliarem a probabilidade de sucesso financeiro de projetos recheados de incertezas [13]. Outra ferramenta tradicional de avaliação de investimentos, como fluxo de caixa descontado, é adequado quando os eventos de fluxos de caixa futuros são determinísticos, porém é limitado quando há muita incerteza sobre o negócio, pois falham em fornecer uma avaliação sólida sobre um ambiente incerto e quando existe flexibilidade nas decisões gerenciais, neste cenário RO é adequado [14]. A indústria de nanomateriais é caracterizada por processos inovadores em todas as fases do ciclo do produto e a análise financeira convencional torna qualquer projeção incerta e com alto grau de risco. O modelo que combina vários métodos analíticos, incluindo uma forma recursiva de Custeio do Ciclo de Vida (LCC), bem como simulação de Monte Carlo e RO, apresentam vantagem para inovar [15].

Enquanto inovações incrementais são fundamentadas por dados históricos e tecnologias conhecidas, a RI é caracterizada por incertezas e, por esta razão, técnicas financeiras tradicionais como VPL e retorno sobre investimento (ROI) não são adequadas para projetos de RI, pois não são capazes de lidar com falta de dados históricos e incertezas. RO é considerado por alguns autores como uma abordagem adequada ao contexto de RI [8].

O gerenciamento eficaz do portfólio de projetos de pesquisa e desenvolvimento (P\&D) é essencial para a produtividade de uma empresa, capacidade de inovação e para promover sua sobrevivência [16]. A abordagem RO para avaliação destes projetos tem a vantagem de lidar com riscos e contribuir com tomadas de decisão estratégicas para o gerenciamento sustentável [16]. O método de avaliação com o 
modelo de rede binomial de reversão de média sob o regime de Markov (MRBL-MRS) pode considerar os riscos de mercado através da aplicação de parâmetros, tais como volatilidade e taxas de juros livres de risco [16]. Projetos de investimentos em estágios múltiplos estão sujeitos a muitas incertezas e apresentam flexibilidades durante e depois do processo de desenvolvimento. RO pode prover uma maneira eficaz para avaliar projetos de P\&D, fornecendo precisão adequada quando comparado a outros modelos de avaliação de investimentos [17].

A principal vantagem da abordagem fuzzy é o processo especialista para trabalhar com incertezas e opiniões subjetivas [18]. O autor realizou uma pesquisa com 91 pequenas e médias empresas (PMEs) do México e apresentou uma estrutura metodológica para mensurar a gestão da inovação através da abordagem fuzzy. Projetos de parcerias público-privadas (PPP), promove inovação e implementa novas tecnologias. Pesquisas anteriores demonstraram que RO empregadas durante $\mathrm{O}$ processo de formalização de PPP podem mitigar estes riscos [19]. Políticas de avaliação de inovação são instrumentos que impedem o potencial fracasso no mercado de uma mudança tecnológica radical e também visa proteger os espaços de incubação de inovações e organizar sua difusão [20].

Percebe-se que RO foi o mais indicado, nos artigos qualificados, como aplicável à avaliação de projetos de RI ou de grandes incertezas. Segue distribuição destas indicações: RO (64\%), todas as 05 outras indicadas, somadas, representam 36\%, são elas: políticas de avaliação, MRBL-MRS, fuzzy, monte carlo e LCC.

\subsection{Distribuição das Publicações por Ano e Categoria}

Todas as 11 publicações qualificadas são da categoria Journal Article, sendo 07 da base de dados Scopus, correspondendo a $64 \%$ do total. Segue distribuição destes artigos por ano de publicação:

- 2017: 03 artigos [15,18,19], sendo todos da base Scopus.

- 2018: 06 artigos, sendo 03 da base de dados Scopus [13-15] e 03 da base de dados Web of Science $[8,16,17]$.

- 2019: 02 artigos, sendo o [12] da base de dados Scopus e [11] da base Web of Science.

\subsection{Distribuição dos Artigos por Pontuação Média}

Dos 11 artigos analisados, 08 obtiveram pontuação média igual a 3, correspondendo a $73 \%$ dos artigos qualificados, e $27 \%$ foram publicados no Sustainability (Switzerland). Segue relação e pontuação média respectiva:

- $\mathrm{PM}=3$ : total de 08 artigos [8,11-17]

- $\mathrm{PM}=2$ : total de 01 artigo [18]

- $P M=1,5:$ total de 02 artigos $[19,20]$

- $\mathrm{PM}=1$ : total de 01 artigo [21] descartado. 


\section{CONCLUSÃO}

Este trabalho teve como objetivo caracterizar o conhecimento atual sobre quais ferramentas e técnicas de avaliação de investimentos são aplicáveis à avaliação de projetos de inovação radical ou de grandes incertezas.

As 11 publicações qualificadas para extração e análise de dados de fato estavam bem alinhadas ao propósito desta revisão integrativa de literatura, ou seja, continham informações sobre ferramentas e técnicas de avaliação de investimentos aplicáveis à avaliação de projetos de inovação radical ou de grandes incertezas.

Técnicas e ferramentas tradicionais de avaliação de investimentos como, por exemplo, VPL, ROI e fluxo de caixa descontados não foram indicadas, por estas publicações, para avaliação de projetos de inovação radical que, devido à ausência de dados históricos e ambiguidades do mercado, são caracterizados por alto grau de incertezas.

$\mathrm{RO}$ tem sido apresentada nas literaturas recentes como de grande utilidade para a mensuração de inovações, principalmente quando o grau de incerteza é alto, além de servir de apoio às tomadas de decisão quanto à alocação de investimentos em projetos desta natureza. Outro fator importante para reduzir os riscos de investimentos em projetos de grandes incertezas é a necessidade de conhecer bem o mercado e atuar de forma a perceber e a criar novos valores para sociedade e, neste quesito, políticas são vetores de mudanças.

Devido às restrições de formatação e número de páginas deste modelo de artigo, esta revisão integrativa de literatura não explorou de forma detalhada as técnicas e ferramentas apresentadas como adequadas à avaliação de projetos de inovação radical ou de grandes incertezas. Por outro lado, esta limitação oferece oportunidade para 0 desenvolvimento de outros trabalhos relacionados ao detalhamento e aplicação destas técnicas e ferramentas, principalmente a RO que foi a mais mencionada nos artigos qualificados para a elaboração deste trabalho.

\section{REFERÊNCIAS}

[1] LIANTO, Benny; DACHYAR, M.; SOEMARDI, Tresna Priyana. Continuous innovation: a literature review and future perspective. International Journal on Advanced Science, Engineering and Information Technology, v. 8, n. 3, p. 771779, 2018.

[2] NISULA, Anna-Maija; KIANTO, Aino. Evaluating and developing innovation capabilities with a structured method. Interdisciplinary Journal of Information, Knowledge, and Management, v. 8, p. 59-82, 2013.

[3] CHRISTENSEN, Clayton M.; RAYNOR, Michael E.; MCDONALD, Rory. What is disruptive innovation. Harvard Business Review, v. 93, n. 12, p. 44-53, 2015. 
[4] CHRISTENSEN, Clayton M. O Dilema da Inovação: quando as novas tecnologias levam empresas ao fracasso. São Paulo: M. Books, 2012.

[5] PMI - Project Management Institute. The Standard for Portfolio Management. Newton Square - Pennsylvania, 2006.

[6] RIBEIRO, R. et al. From idea to benefit: Project portfolio management using front end loading, the standard for portfolio management and PRINCE2. International Journal of Management (IJM), v. 4, n. 5, p. 60-68, 2013.

[7] SILVA, A. et. al. Value management in IT projects: a study in Brazilian organizations. Gestão \& Produção, 2019. Disponível em: <https://doi.org/10.1590/0104-530X-3064-19>. Acesso em: 27 Jul. 2019

[8] BRASIL, V. et. al. Valuation of innovation projects with high uncertainty: Reasons behind the search for real options. Journal of Engineering and Technology Management - JET-M, v.49, p.109-122, 2018.

[9] PAULSON, Albert S.; O'CONNOR, Gina Colarelli; ROBESON, Daniel. Evaluating radical innovation portfolios. Research-Technology Management, v. 50, n. 5, p. 1729, 2007.

[10] HENRIETTE, Emily; FEKI, Mondher; BOUGHZALA, Imed. The shape of digital transformation: a systematic literature review. MCIS 2015 Proceedings, p. 431-443, 2015.

[11] TARIFA-FERNÁNDEZ, Jorge; SÁNCHEZ-PÉREZ, Ana María; CRUZ-RAMBAUD, Salvador. Internet of Things and Their Coming Perspectives: A Real Options Approach. Sustainability, v. 11, n. 11, p. 3178, 2019.

[12] ARANDA, Fernando Cruz; BUSTAMANTE, Antonia Terán. Valuation of an investment project in research and development in the pharmaceutical industry. Contaduría y Administración, v.64, n.1, p. 90, 2018.

[13] HERNANDEZ-GARCIA, Daniel; GUEMES-CASTORENA, David; JARAMILLO, Idalia Ponce. A real option based model for the valuation of patent protected technological innovation projects. World Patent Information, v.53, p. 24-38, 2018.

[14] KIM, Sungchul; GIACHETTI, Ronald; PARK, Sangsung. Real options analysis for acquisition of new technology: A case study of Korea K2 Tank's Powerpack. Sustainability (Switzerland), v.10, n.11, 2018.

[15] VLACHY, J. The value of innovation in nanotechnology. Engineering Economics, v.28, n.5, p. 535-541, 2017.

[16] PARK, Jung; SHIN, Kwangsoo. R \& D project valuation considering changes of economic environment: A case of a pharmaceutical $R$ \& D project. Sustainability (Switzerland), v.10, n.4, 2018. 
[17] BRANDÃO, Luiz E.; FERNANDES, Gláucia; DYER, James S. Valuing multistage investment projects in the pharmaceutical industry. European Journal of Operational Research, v.271, n.2, p. 720-732, 2018.

[18] ALFARO-GARCIA, Victor G.; GIL-LAFUENTE, Anna M.; ALFARO CALDERON, Gerardo G. Kybernetes, v.46, n.1, p.50-56, 2017.

[19] FITCH, G. et. al. System Dynamics Models for the Valuation of Real Options in P3 Concession Project Agreements. Journal of Infrastructure Systems, v.24, n.1, p.04017047, 2017.

[20] HUGUENIN, Ariane; JEANNERAT, Hugues. Creating change through pilot and demonstration projects: Towards a valuation policy approach. Research Policy, v.46, n.3, p. 624-635, 2017.

[21] LOYARTE, E. et. al. Model for calculating the intellectual capital of research centres. Journal of Intellectual Capital, v.19, n.4, p.787-813, 2018. 